\title{
Modeling Of Hot Oil Leakage At Regeneration Gas Heater On Dehydration Process Of Natural Gas Liquified Extraction (NGLE) Gas Plant
}

\author{
Yung Azani ${ }^{1}$, Armila ${ }^{2}$, Rudi Kurniawan Arief ${ }^{3}$ \\ Fakultas Teknik, Prodi Teknik Mesin, Universitas Muhammadiyah Sumatera Barat ${ }^{1,2,3}$ \\ email: agil_loe@yahoo.com ${ }^{1}$, kimmylala74@gmail.com ${ }^{2}$, rudi.arief@gmail.com ${ }^{3}$ \\ DOI: http://dx.doi.org/10.31869/rtj.v5i1.2928
}

\begin{abstract}
The regeneration process in saturated dehydrator after working to drying the gas in the dehydration unit in the Natural Gas Liquified Extraction (NGLE) plant. This process is through the heating dehydrator process by flowing the regeneration gas into the dehydrator slowly (rump up temperature) until it reaches the heating temperature, and then holding the condition. Its condition is in accordance with the engineering design and followed by a rump down temperature which the dehydrator will be cooled down and ready for the dehydration process. This regeneration process works automatically in accordance with the engineering design which runs following the logic control that has been implemented into the Distributed Control System (DCS) in the Control Room. All order in DCS to obtain gas that has been moisture limited value which is allowed to be extracted. Regeneration gas was taken from the heat exchange between hot oil and regeneration gas in the regeneration gas heater package. This operation happend when the rump up temperature leaks the hot oil in the flange fitting of the regeneration gas heater package, its causes oil spillage (engineering design standart operation procedur). Its analysis case assumed the leakage is caused by thermal shock in the fittings of regeneration gas heater package in $2 \%$ hot oil supply. To eliminate the thermal shock, a simulation of new models engineering design is initial by opening of the hot oil supply to the regeneration gas heater was changes with increasing its opening during stand-by conditions from $2 \%$ with a temperature at $45.72^{\circ} \mathrm{C}$ to $5 \%$ with a temperature at $51.61 \mathrm{C}$ in the Distributed Control System (DCS) logic control. The results goals with this implementation are no more hot oil leaks occur in the regeneration gas heater package. New models engineering design is stopping hot oil spillage, and maintaining operational continuity without having to spend money on repairing the regeneration gas heater package. process run in new models of engineering design, and this model becomes the new standard operating in start-up and commissioning plant process.
\end{abstract}

\section{Keywords: engineering design, rump up-down temperature, hot oil, dehydration, leakage 1.}

\section{INTRODUCTION}

\section{Fundamental}

The Natural Gas Liquified Extraction (NGLE) process plant will extract natural gas into methane $(\mathrm{CH} 4)$ and ethane $(\mathrm{C} 2 \mathrm{H} 6)$ gas products. Gas processing operations are supported by a control system called the Distributed Control System (DCS) which provides accuracy, response speed, and manipulation of operational commands that can be carried out by operators in the control room. Dehydration process is the initial process of gas before extraction process, this process is the process of drying the gas by removing the moisture present in the natural gas that occurs in the dehydrator. Adsorption is referred to as the process of drying the gas in a time programmed into the DCS until the dehydrator becomes saturated with moisture and must be regenerated. Regeneration is a dehydrator rehabilitation process by flowing regeneration gas during the heating step of the regeneration process into the dehydrator so that the regeneration gas can carry the moisture absorbed in the molecular sieve away. Regeneration gas is obtained from heat exchange with hot oil in the regeneration gas heater package. At the time of rump up temperature in the heating step there was found a hot oil leak that causes the oil spillage and pollution. Field data collection, operational trend data from DCS in the control room and engineering data are the first steps taken to find the cause of this problem, then modeling is the next step to make it easier to take the hot oil leak hypothesis. In order to keep operations running, changes to the operational logic of dehydration sequence in DCS can be made to stop this leak, this change is in the form of manipulating work orders for openings of hot oil supply temperature control 
valve engineering design so that hot oil leaks can be stopped.

\section{Scope}

In this journal the scope of study is about get new model of engineering design of hot oil supply control valve opening logic control on DCS during standby mode to stop hot oil leakage on regeneration gas heater.

\section{Research goals}

This study aims to find the way how to stop the hot oil leakage at regeneration gas heater package, to stop the hot oil spillage, to avoid plant shutdown and to avoid production deferment and maintaining operational continuity without having to spend money on repairing the regeneration gas heater package.

\section{MeTHODS}

\section{Flow Chart Research}

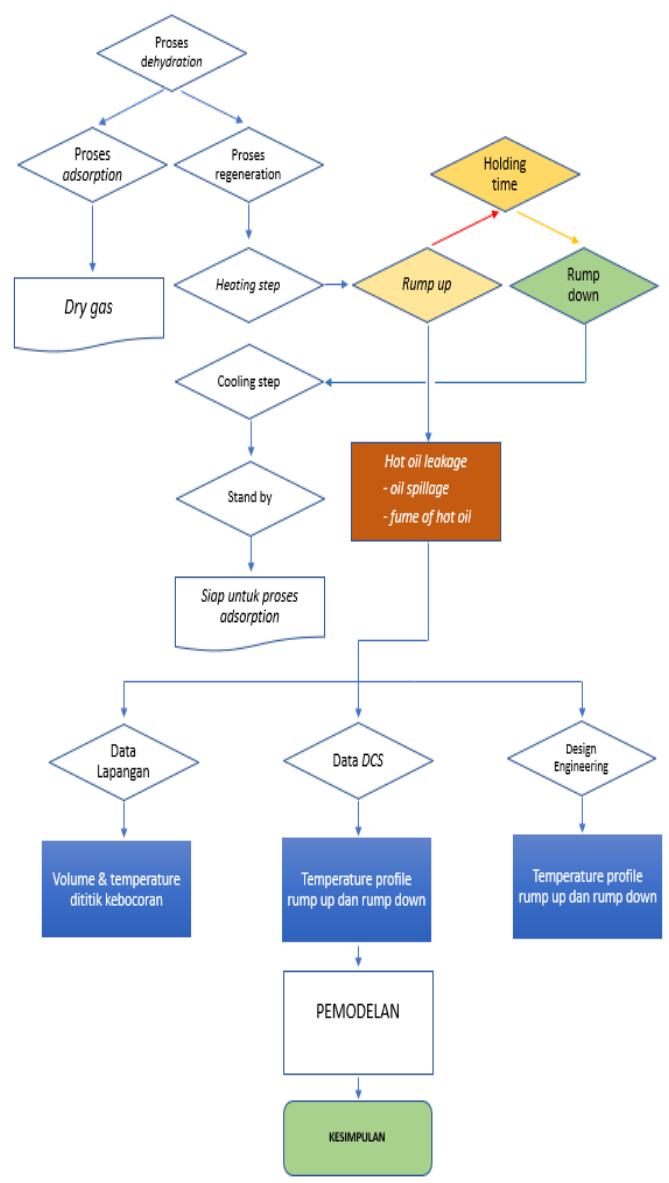

Figure 1. Research flow chart

\section{Dehydration Process}

Dehydration Process is the process of drying gas or removing moisture contained in the gas before it is flowed into the extraction process because this moisture can form the formation of hydrate [9], which causes blockages in the tubing in the gas extraction process, this process occurs in a vessel called a dehydrator vessel. This process can consist of a minimum of 2 dehydrators vessels or more depending on the capacity of the plant process or the percentage of moisture content that must be removed.

The working principle of dehydration is adsorption, which is a gas drying method using a solid medium molecular sieve desiccant which will trap moisture particles [7]. The main steps are adsorption and regeneration.

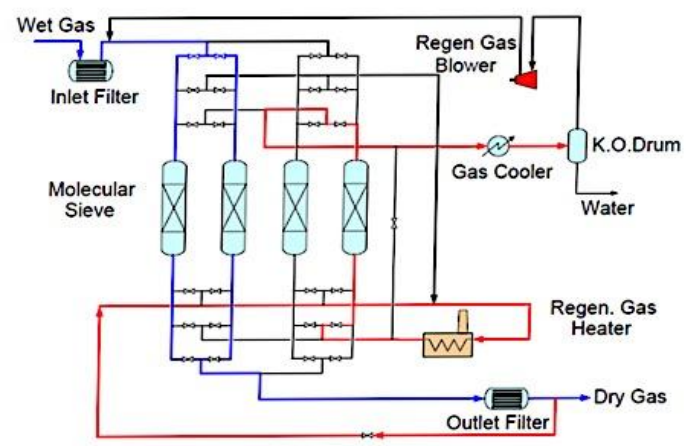

Figure 2.. Simple schematic of dehydration

\section{Process}

A simple schematic for the gas drying process to obtain dry gas can be represented in Figure 2.1. Wet gas or gas supply containing moisture will enter through the incoming pipe and then separation occurs at the inlet of the separator so that the heavy fraction of the gas is separated, it is hoped that only a little moisture will flow into the dehydrator vessel so that it can reduce the burden of the drying process. The dehydrator with the blue line in Figure 2 indicates that the dehydration process is in progress. While the dehydrator vessel with the red line in Figure 2 indicates that the molecular sieve desiccant regeneration process is in progress in the dehydrator vessel with the sequence regulated by the logic control system implemented in the Distributed Control System (DCS) plant process in the control room. This dehydration process runs in parallel.

Regeneration process is the process of rehabilitating molecular sieve desiccant in a dehydrator vessel so that it can be reused to capture moisture. 
The regeneration process is divided into two steps, namely the heating step and the cooling step.

\section{Heating step}

The heating step is the process of flowing regeneration gas, which has been heated in the regeneration gas heater into the dehydrator vessel through the gas outlet of the dehydrator vessel as shown in Figure 3 below. The red line is the regeneration gas can carry the regeneration gas flow that flows from the bottom to the top of the dehydrator vessel with the aim that the moisture trapped in the molecular sieve desiccants away.

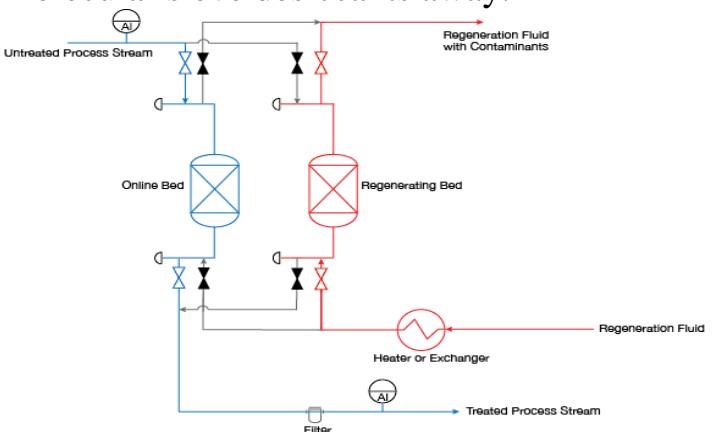

Figure 3 Adsorption process and regeneration process of dehydrator vessel

\section{Cooling Step}

Cooling step is the process of cooling the dehydrator vessel by flowing the regeneration gas that is not heated in the regeneration gas heater to the dehydrator vessel where the red line is the direction of the regeneration gas flow that is not heated again in the regeneration gas heater. Its purpose is to cool the dehydrator vessel so that it is ready to be reused for gas drying.

The regeneration temperature profile [8] is shown in Figure 4 steps during ramp-up are included to prevent overheating, which could result in the formation of hot moisture.

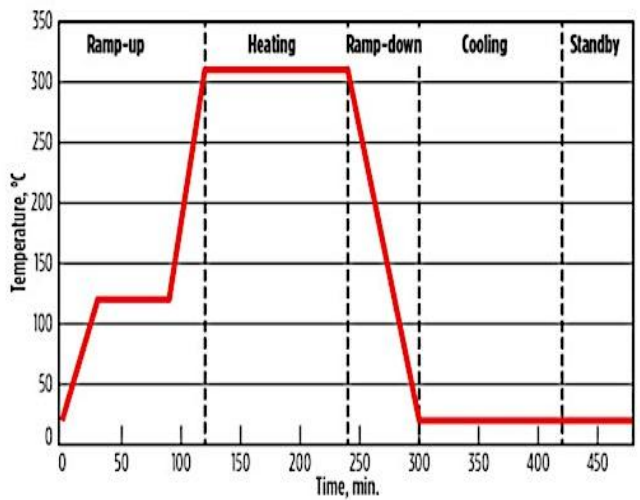

Figure 4 Dehydration process temperature profile

\section{Regeneration Gas Heater Package}

Regeneration gas heater package is a package for heat exchange, this package are consists of a shell and tube, made of one tube or a series of parallel tubes (tube bundles) enclosed in a cylindrical pressure vessel as shown in Figure 5 below.

The design of the device is such that one liquid flows through the smaller tube and the other flows around the outside in a closed housing.

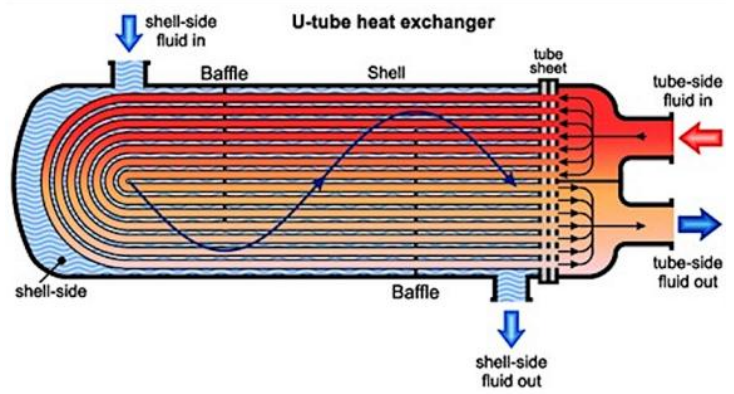

Figure 5 Part of the regeneration gas heater package

The regeneration gas heater package consists of several parts with the following explanations:

\section{Shell Side}

Hot oil Regeneration gas will flow to the shell side in the regeneration gas heater package with an operating pressure around 60 Barg and a temperature of $-29 / 340{ }^{\circ} \mathrm{C}$, the shell side is made of steel that has been designed to withstand high pressure and temperature as shown in Figure 6.

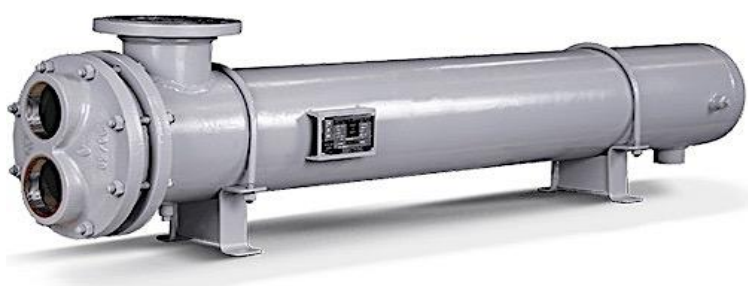

Figure 6 Shell side body

Tube Side

Regeneration gas flows with a design pressure of 75 Barg and a temperature of -29 / $316^{\circ} \mathrm{C}$. The tube is made of bronze because it has a good heat conductor in addition to a low 
price, and is corrosion resistant [3] as shown in Figure 7.

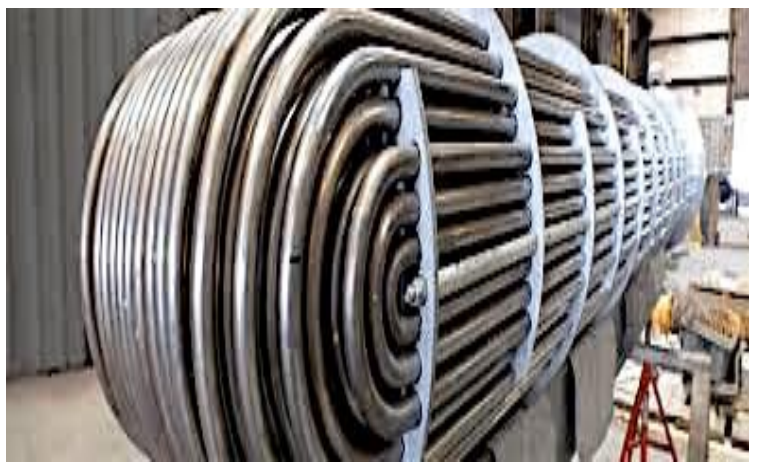

Figure 7 Tube side assembly

\section{Gasket}

The gasket used for all flanges is a type of soft iron spiral wound gasket with a code of 316L Stainless Steel part number PN 29AE002-41-519 with a diameter of 6 inches, schedule $80 \mathrm{~mm}$ and a pressure rating of 600 psi which works at a temperature of $-100 / 550^{\circ}$ $\mathrm{C}$ with a pressure of $250 \mathrm{Barg}$.

The material construction of spiral wound gaskets is based on the required operating conditions such as temperature, pressure and the fluid acting on the tool. A standard spiral wound gasket can be made of a ring with an inner Stainless Steel (SS) 316 and a winding with graphite filler and carbon steel outer.

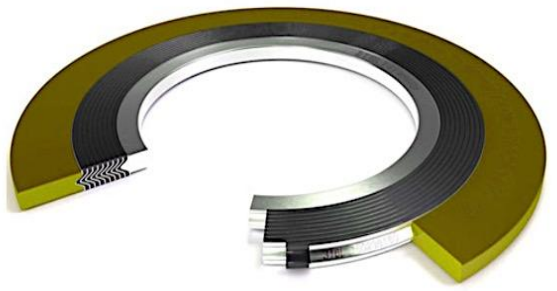

Figure 8 Spiral wound gasket

\section{Bolts and Nuts}

Torque locking bolts and nuts on channel head closures hot oil with a minimum torque of $680 \mathrm{~N} . \mathrm{m}$ and a maximum of $1220 \mathrm{Nm}$. The uneven locking torque will affect the sealing system fitting in the regeneration gas heater package.

\section{Hot Oil}

The heating medium is hot oil with the name Therminol 66, the operating design for the heating medium is $310-320^{\circ} \mathrm{C}$ (table 2.4).
Hot oil is obtained through the exchange temperature exhaust gas turbine in the Waste Heat Recovery Unit (WHRU) [1] with a temperature of $360{ }^{\circ} \mathrm{C}$.

\section{Distributed Control System (DCS)}

Distributed Control System or DCS is a platform for a system with automatic control and operation in industrial processes. DCS is the brain of the control system. DCS combines and integrates Human Machine Interface (HMI), controller, historian, database and alarm management into a single automated system. DCS was created to control several separate controllers that work on various continuous operations. Everything is connected using a high-speed communication protocol.

DCS is composed of several parts or devices. Some of them are central computers, public computers, local computers or controllers, displays, databases and data lines. The central computer is the main computer that is connected to everything related to the operating system. General computer is a control unit used to perform optimization, advanced settings and so on. The local computer is used to directly control a process, which is usually, based on Proportional Integral Derivative (PID). Display serves to display a graph or analysis of a process. The database serves to store and collect data. And finally, the data line is a digital data transmission channel that connects all devices in the system. Below the DCS graphic for dehydration proses as shown in Figure 9.

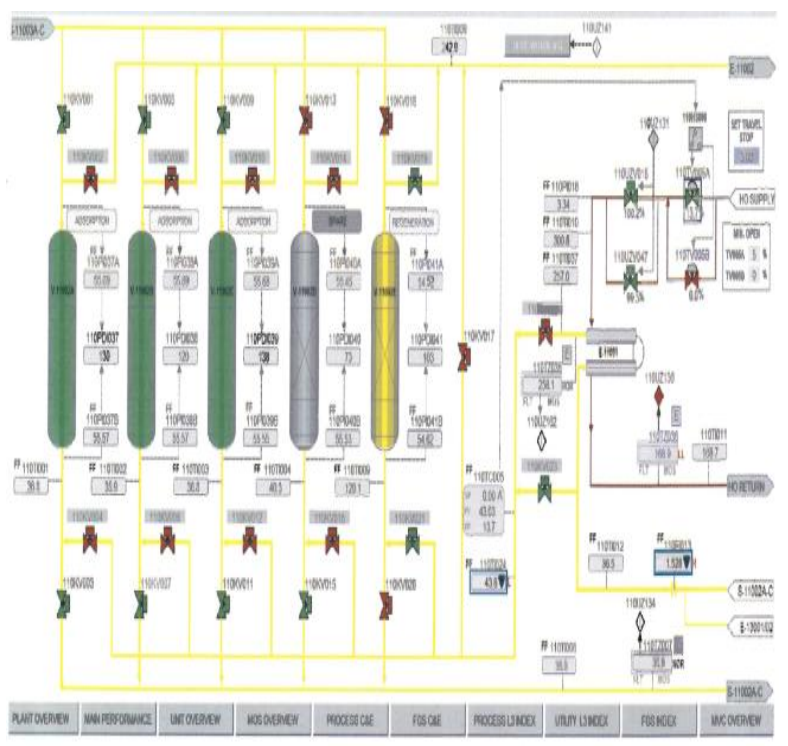

Figure 9 DCS graphic for dehydration 


\section{RESULT}

\section{Hot Oil Leakage Data Field \\ Observation}

Base on field data observation below was mentioned during rump up temperature on heating step with design engineering data the hot oil leakage was occur with the biggest drop at the time cycle 45 minutes and total hot oil leakage was 247 drop per minutes.

Table 1. Leakage volume during heating step (drop/min) observation data design

Engineering

\begin{tabular}{|c|c|c|c|}
\hline $\begin{array}{c}\text { TIM } \\
\text { E }\end{array}$ & $\begin{array}{c}\text { TEMPERATU } \\
\text { RE DITITIK } \\
\text { OBSV. D }\left({ }^{\circ} \mathbf{C}\right)\end{array}$ & $\begin{array}{c}\text { HO } \\
\text { S \% }\end{array}$ & $\begin{array}{c}\text { LEAKAG } \\
\boldsymbol{E} \\
(\text { drop/min } \\
)\end{array}$ \\
\hline $\mathbf{1}$ & 78 & 2 & 0 \\
\hline $\mathbf{5}$ & 97 & 8 & 0 \\
\hline $\mathbf{1 0}$ & 120 & 15 & 3 \\
\hline $\mathbf{1 5}$ & 136 & 22 & 24 \\
\hline $\mathbf{2 5}$ & 157 & 28 & 33 \\
\hline $\mathbf{3 0}$ & 168 & 32 & 53 \\
\hline $\mathbf{4 5}$ & 190 & 41 & 69 \\
\hline $\mathbf{6 0}$ & 229 & 47 & 40 \\
\hline $\mathbf{1 2 0}$ & 249 & 53 & 25 \\
\hline
\end{tabular}

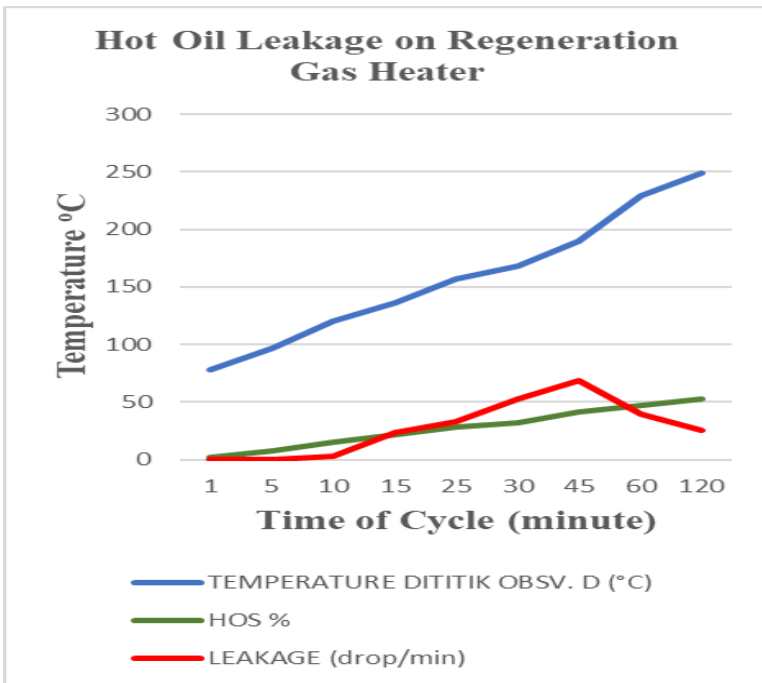

Figure 10 Graph of leakage during heating step (drop/min) observation data design engineering

After new input on hot oil supply valve opening from $2 \%$ into $5 \%$, we will get data as below table 1 and graph 2 .
Table 2. Leakage volume during heating step (drop/min) observation data design engineering

\begin{tabular}{|c|c|c|c|}
\hline $\begin{array}{c}\text { TIM } \\
\text { E }\end{array}$ & $\begin{array}{c}\text { TEMPERATUR } \\
\text { E DITITIK } \\
\text { OBSV. D }\left({ }^{\circ} \mathbf{C}\right)\end{array}$ & $\begin{array}{c}\text { HO } \\
\text { S } \\
\text { \% }\end{array}$ & $\begin{array}{c}\text { LEAKAG } \\
\boldsymbol{E} \\
(\text { drop/min } \\
)\end{array}$ \\
\hline $\mathbf{1}$ & 97 & 5 & 0 \\
\hline $\mathbf{5}$ & 120 & 15 & 0 \\
\hline $\mathbf{1 0}$ & 136 & 21 & 0 \\
\hline $\mathbf{1 5}$ & 157 & 26 & 0 \\
\hline $\mathbf{2 5}$ & 168 & 31 & 0 \\
\hline $\mathbf{3 0}$ & 190 & 36 & 0 \\
\hline $\mathbf{4 5}$ & 229 & 42 & 0 \\
\hline $\mathbf{6 0}$ & 249 & 47 & 0 \\
\hline $\mathbf{1 2 0}$ & 249 & 51 & 0 \\
\hline
\end{tabular}

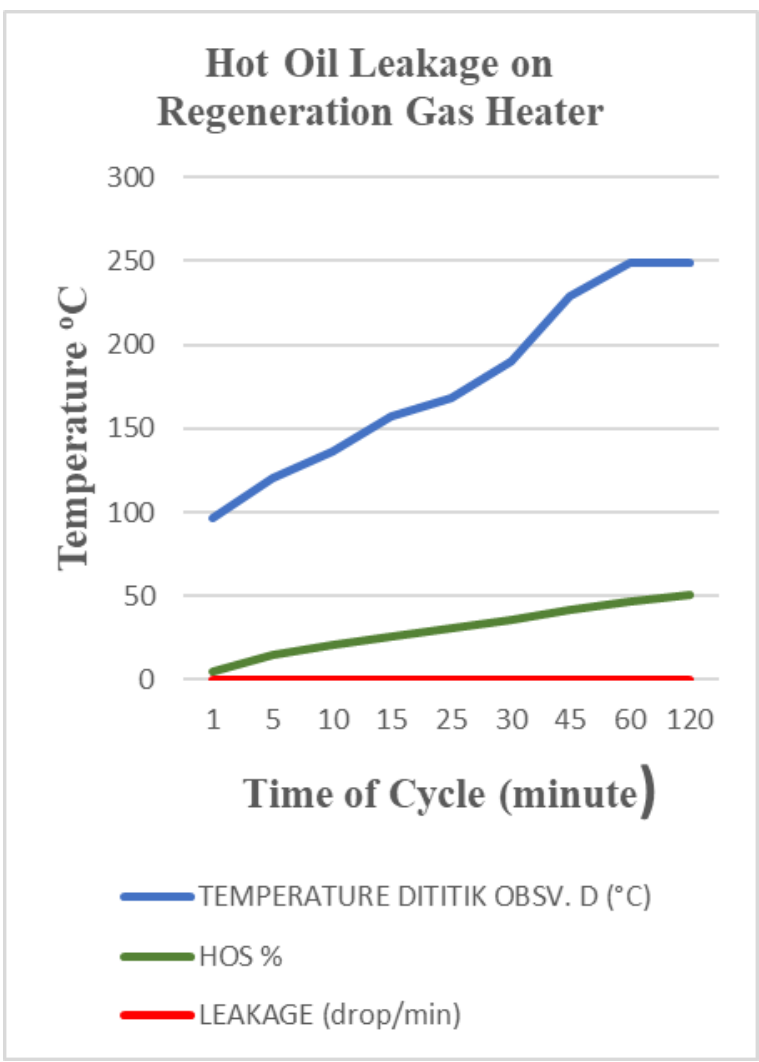

Figure 11 Graph of leakage during heating step (drop/min) observation new input on hot oil supply valve opening from $2 \%$ into $5 \%$ 
Base on field data observation above was mentioned during rump up temperature on heating step with manipulating on hot oil supply valve opening from $2 \%$ into $5 \%$ there is no hot oil leakage anymore.

\section{Temperature Profile Design}

Engineering VS Temperature Profile Hot Oil Supply Opening 5\%

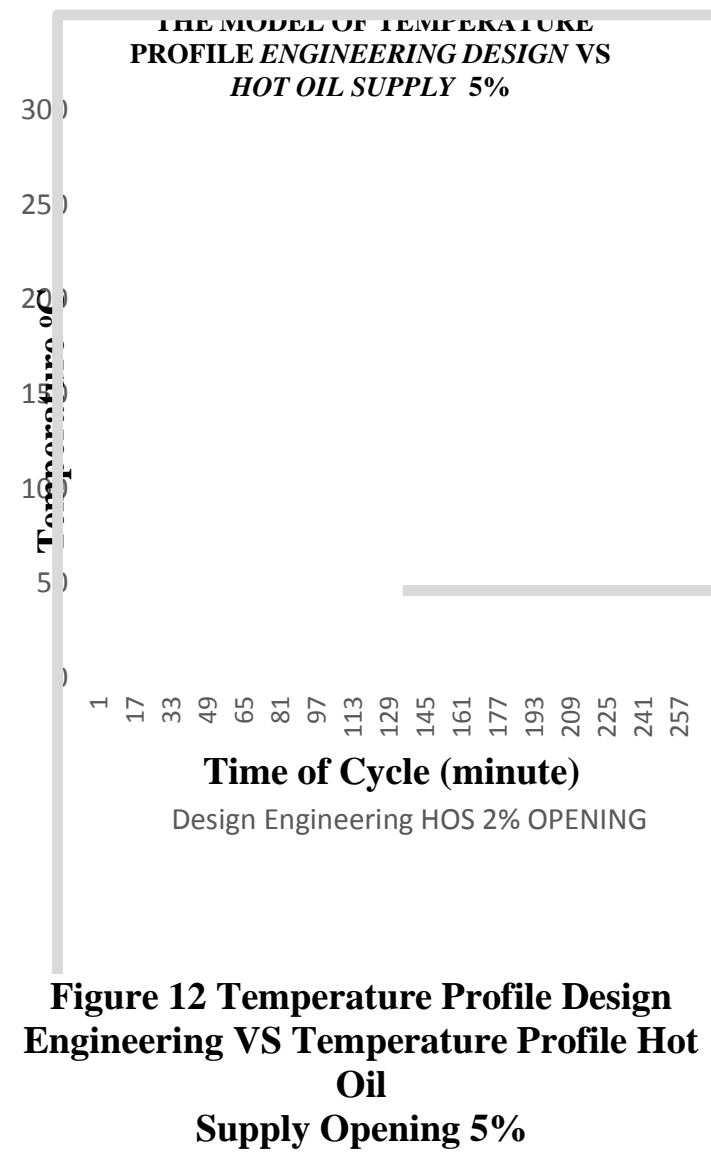

From the modeling curve of Figure 12 the difference between design engineering vs HOS $5 \%$ is as follows:

1.The initial temperature when starting the heating step is not the same.

2. Rump up temperature is not the same.

3.In the engineering design there is no rump down temperature, at 5\% hot oil supply there is a rump down temperature.

\section{CONCLUSION}

The hypothesis of hot oil leakage in the hot oil of the Regeneration Gas Heater occurs in the shell side fitting and the tube side fitting happen due to thermal shock on fitting flanges.
By new model input of engineering design the value on hot oil supply valve opening from $2 \%$ into $5 \%$ this has increased the initial heating step temperature, reduced thermal shock on the sealing part in the shell side fitting and tube side fitting hot oil inlet regeneration gas heater so as to stop the hot oil leak.

The DCS trend has shown that the operational temperature profile is getting better after changing the hot oil supply valve opening to the regeneration gas heater.

\section{REFERENCES}

Journal:

1. Mitra, S. (2015). Design Considerations of Hot Oil System. New Castle, England.

2. Ghinna Mahassin, M. A. (2021). Mengenal Distributed Control System (DCS).

3. Runquillo, R. (2021). Understanding Heat Exchangers. North America: THOMAS INDUSTRY MANUFACTURE.

4. Tom Lessard, P. S. (2018). Improve Regeneration of Adsorbent Beds. New York: AICHE.

5. Michal Netsul, P. D. (2011). Natural Gas Extraction to End. United Kingdom: IntechOpen Chemical Engineering.

6. Willmott. (2011). Regenerative Heat Exchangers . London: Thermopedia.

7. Myrlla G. R.S, L. M. (2017). Natural Gas Dehydration by Molecular Sieve in Offshore Plants. Brazil: ELSEVIER SCIENCEDIRECT.

8. Mokhatab, S. Optimal Design adn Operation of Molecular Sieve Gas Dehydration Units. (R. H. Herold, Ed.) Halifax, Nova Scotia, Canada: Gulf Publishing Holdings LLC.

9. Marko Jaric, S. P. (2020). Inspection adn Repair Quality Plan of Regeneration Gas Heater. (D. N. Sirbu, Ed.) Chicago: Thans Tech Publications Ltd.

\section{Textbooks:}

1. Construction, G. E. (2018). Operating Manual - NGLE Facilities (Dehydration). Korea: GS E\&C.

2. Construction, G. E. (2018). Plant Pipeline Operating Procedure and Manual. Korea: GS.

3. SA, B. F. (2018). Genaral Arrangement Drawing E-11001 Regenaration Gas Heater. Korea: GS. Engineering, G. C. 
(2019). Control Narrative NGLE Plant.

Korea: GS.

4. Kamalogis Teknik Fisika .Yokogawa. (2021). Distributed Control System (DCS).

Yokogawa. 